\title{
Impact of transcatheter aortic valve implantation in patients with reduced ejection fraction
}

\author{
Hüseyin Ayhan ${ }^{1}$, Hacı Ahmet Kasapkara ${ }^{1}$, Tahir Durmaz ${ }^{1}$, Telat Keleş ${ }^{1}$, \\ Abdullah Nabi Aslan², Cenk Sarı ${ }^{2}$, Serdal Baştuğ ${ }^{2}$, Emine Bilen ${ }^{2}$, \\ Nihal Akar Bayram ${ }^{1}$, Murat Akçay ${ }^{1}$, Engin Bozkurt ${ }^{1}$ \\ ${ }^{1}$ Department of Cardiology, Faculty of Medicine, Yıldırım Beyazıt University, Turkey \\ ${ }^{2}$ Department of Cardiology, Ankara Ataturk Education and Research Hospital, Turkey
}

\begin{abstract}
Background: Aortic stenosis increases with age. According to guidelines, left ventricular systolic dysfunction is an indication for aortic valve replacement, even in asymptomatic patients. There is no clear data on the application of transcatheter aortic valve implantation (TAVI), which is a method showing continuous improvement in recent years, in patients with reduced ejection fraction (REF) having a poor prognosis for surgical aortic valve replacement. We therefore aimed to investigate the effect of TAVI on left ventricular ejection fraction (LVEF) and also its efficacy and safety in patients with REF.

Methods and results: The study included 104 patients who underwent transfemoral TAVI in our clinic. The patients were divided into two groups: $L V E F \leq 45 \%$ (REF group, $n=28$ ) and $L V E F>45 \%$ (preserved ejection fraction [PEF] group, $n=76$ ). Follow-up measurements were performed at baseline, discharge, $1^{\text {st }}, 6^{\text {th }}$ and $12^{\text {th }}$ months. No statistical difference was found between the groups with respect to complications and mortality rates. A statistically significant difference was detected in LVEF after TAVI, either in all patients (53.9 \pm 14.6 , $57.0 \pm 11.4,59.4 \pm 8.4,60.4 \pm 6.8,63.2 \pm 3.9$, respectively, at baseline, discharge, $1^{\text {st }}, 6^{\text {th }}$ and $12^{\text {th }}$ months, $\left.p<0.001\right)$ or in the groups separately. A statistically significant increase in LVEF $(p<0.001)$ was determined at discharge, $1^{\text {st }}, 6^{\text {th }}$ and $12^{\text {th }}$ months, whereas LVEF increased in all follow-ups of the PEF group, however this elevation reached a statistical significance only at the $1^{\text {st }}$ month $(p=0.04)$.
\end{abstract}

Conclusions: Our study has shown the positive effect of TAVI on LVEF and its effective and safe applicability in patients with REF. (Cardiol J 2015; 22, 1: 108-114)

Key words: transcatheter aortic valve implantation, reduced ejection fraction, heart failure

\section{Introduction}

Severe aortic stenosis (AS) is a common valvular heart disease that leads to a high morbidity and mortality rate. Aortic valve replacement (AVR) is recommended in patients with severe AS that are symptomatic or asymptomatic accompanied by left ventricular ejection fraction (LVEF) $<50 \%$ [1]. Surgical replacement of the narrowed aortic valve (s-AVR) is performed with a low operative morta-

Address for correspondence: Hüseyin Ayhan, MD, Ankara Ataturk Education and Research Hospital, Department of Cardiology, Street: Bilkent, Postal code: 06800, Ankara, Turkey, tel: +90 3122912525, fax: +90 3122912745, e-mail: huseyinayhan44@yahoo.com

Received: 31.03.2014 Accepted: 17.04.2014 
lity rate in the absence of severe co-morbid conditions. Patients given s-AVR show both improved symptoms and prolonged survival. Nevertheless, s-AVR cannot be applied in $30 \%$ of patients because of left ventricular dysfunction, advanced age, or co-morbid conditions [2, 3]. Since s-AVR shows a high pre-operative risk, a conservative approach is preferred in patients with LVEF (reduced ejection fraction $[R E F]$ ). S-AVR is also associated with operative mortality in patients with severe AS and $\operatorname{REF}[4,5]$. Transcatheter aortic valve implantation (TAVI) is an effective method for patients with severe AS that present a higher surgical risk or who cannot undergo s-AVR [6, 7]. Some studies have shown an improved effect of TAVI on LVEF [8]. However, the efficacy and safety of TAVI in patients with REF is not clearly proven. Consequently, guidelines accept LVEF $<20 \%$ as a relative contraindication for TAVI [1]. We have also aimed in our study to investigate the effect of TAVI on left ventricular function in addition to its applicability, efficacy, and safety in patients with REF.

\section{Methods}

Between July 2011 and July 2013, 104 patients who were inoperable or at high risk for s-AVR due to severe calcified AS and co-morbid conditions were selected for TAVI in our clinic. The patients were divided into two groups, the group with preserved LVEF (PEF) and the group with reduced LVEF (REF). The REF group included a total of 28 patients. Five patients in the REF group were in the low-flow/low-gradient class (mean gradient $<40 \mathrm{~mm} \mathrm{Hg}$ ). The diagnosis of severe AS was confirmed following dobutamine stress echocardiography (DSE) performed with these patients. Transthoracic echocardiography (TTE) Doppler and 2-dimensional images were obtained from parasternal long and short axis, apical 4-chamber, and subcostal 4-chamber images. TTE was reviewed to assess the pericardium, valvular anatomy and function, and cardiac function. All patients underwent multi-slice computed tomography and transesophageal echocardiography before the procedure. Valve morphology, aortic annulus, coronary ostium-annulus distance, calcification, suitability of peripheral arteries, and the possibility of an additional pathology were also analyzed. Echocardiography (Philips iE33) was performed prior to TAVI and at hospital discharge, $1^{\text {st }}, 6^{\text {th }}$ and $12^{\text {th }}$ months follow-ups. Doppler echocardiographic measurements included left ventricular end-diastolic volume, where LVEF was calculated with the
Simpson method, transvalvular pressure gradient was determined by the Bernoulli formula, and aortic valve area was calculated by the continuity equation. All echocardiography parameters were evaluated according to guidelines of the American Society of Echocardiography [9]. Echocardiography evaluations were performed by a single author. This is our limitation but intra-observer variability was evaluated by performing re-measurements in 15 patients. Intra-observer variability was found to be $5.5 \%$ by echocardiographic measurements. Because of these patients have frailty we could not use the other methods for measurement of LVEF like magnetic resonance imaging or nuclear scintigraphy.

TAVI was performed through the subclavian artery, via a supra-aortic approach and by transfemoral access in 5, 1 , and 98 patients, respectively. TAVI was performed under general anesthesia in 5 and 12 patients from the REF group and PEF group, respectively, while all other patients were given local anesthesia. A vascular closure device was used in 77 patients while surgical closure was used in the remaining patients. Patients were heparinized to achieve an activated clotting time of 250-300 s. TAVI was performed using Edwards Sapien XT (Edwards Lifesciences, Irvine, CA, USA) balloon-expandable prostheses. Three valve sizes of 23 , 26 , and $29 \mathrm{~mm}$ expanded diameter were available. As a result, the cardiac surgery team agreed to apply TAVI. All patients were informed before performing the procedure with the approval of the Ethics Committee of our hospital. Stable patients were discharged and follow-ups at the $1^{\text {st }}, 6^{\text {th }}$ and $12^{\text {th }}$ months were planned. During the follow-ups, routine physical examinations, echocardiography and functional capacities were evaluated.

\section{Statistical analysis}

All analyses were performed using SPSS Statistics Version 17.0. Continuous variables are presented as mean and standard deviation and were compared by means of a 2 -sided Student's t-test. Categorical data are expressed as frequency (percentages) and compared using the $\chi^{2}$ and Fisher's exact tests. Echocardiography data obtained at baseline, discharge, $1^{\text {st }}, 6^{\text {th }}$ and $12^{\text {th }}$ months were compared by repeated measures ANOVA. Continuous variables were compared between patients before and after TAVI using the paired Student's t-test (for normally distributed variables) or the Wilcoxon test (for non-normally distributed variables). Significance was accepted as $\mathrm{p}<0.05$. 


\section{Results}

Of the 104 patients, 69 were female and their mean age was 78.2 years. Among the patients, 28 (26.9\%) were in the REF group and the rest were in the PEF group. The mean valve area was $0.62 \mathrm{~cm}^{2}$ and the average mean gradient was $52.5 \mathrm{~mm} \mathrm{Hg}$ by echocardiography. Mean and maximal gradients were statistically significantly lower in the REF group (mean gradient values of REF and PEF groups were, respectively, $46.9 \pm 13.7$ and $54.6 \pm$ $\pm 13.2, \mathrm{p}=0.01$ ). Of the patients in the REF group, $64.2 \%$ had LVEF $\leq 35 \%$. Also 4 of these patients had LVEF $\leq 20 \%$. Of the patients with LVEF $\leq 35 \%$, only 5 were in the low-flow/low-gradient class. DSE performed in these patients revealed contractile reserve.

The mean Society of Thoracic Surgeons (STS) score of the patients was 7.4 and the mean logistic EUROScore values of the moderate and high risk groups were, respectively, $22.7 \%$ and $90.3 \%$ according to the SURTAVI risk model. STS $(\mathrm{p}<0.001)$ and logistic EUROScore $(p=0.002)$ were found to be statistically significantly higher in the REF group than the PEF group, as expected. Similarly, pulmonary artery systolic pressure was statistically significantly higher $(\mathrm{p}=0.04)$ while LVEF was lower $(\mathrm{p}<0.001)$ in the REF group. Although the REF group had a severe level of chronic obstructive pulmonary disease, no other difference was found between the groups with respect to basal characteristics (age, gender, body mass index, New York Heart Association [NYHA] functional class, prevalence of diabetes or arterial hypertension, history of prior myocardial infarction or stroke, previous coronary artery bypass grafting, or prevalence of atrial fibrillation). The basal characteristics of the patients and TAVI data are shown in Table 1.

TAVI could be performed with a $100 \%$ procedural success. However, a second valve was used in 2 patients due to lower localization in 1 patient and valve embolization in the ascending aorta. After the procedure, a total of 5 intra-hospital mortalities were encountered because of right ventricular rupture due to rapid pacing (in 2 patients), left ventricular rupture due to wire in the left ventricle, postoperative bleeding via supra-aortic approach and left main coronary artery obstruction due to aortic cusp calcification. All of these 5 intra-hospital mortalities were in the PEF group. The follow-ups of the patients revealed mortalities in 2,4 and 5 patients at respectively the $1^{\text {st }}, 6^{\text {th }}$ and $12^{\text {th }}$ months, resulting in a total of $16(15.3 \%)$ mortalities. Mortality rates of both groups were similar.
Echocardiographic follow-up was available for $95 \%$ and $85 \%$ of patients at discharge and at the $12^{\text {th }}$ month, respectively. A statistically significant improvement was found in valve function (mean gradient, aortic valve area) at discharge and at follow-up after TAVI. Severe paravalvular aortic regurgitation developed in none of the patients after TAVI or in follow-ups. Both groups had similar rates of $>+2$ AR detected by TTE $(15 \%$ vs. $24 \%, \mathrm{p}=0.29)$. There were no strokes in the hospital. Only 4 (3.8\%) patients needed permanent pacemaker implantation because of atrioventricular block. Mean hospital stay was $7.3 \pm 5.3$ days and no difference was found between the groups $(8.8 \pm$ \pm 7.1 days in the REF group, $6.8 \pm 4.4$ days in the PEF group; $p=0.09$ ). At the follow-up, $92.8 \%$ and $92.9 \%$ of patients in the REF and PEF groups were found in class I and class II according to NYHA, respectively. An improvement in functional capacity proceeded during the $1^{\text {st }}$ month follow-up (Fig. 1). No difference was found between the groups with respect to complications.

When the effect of TAVI on left ventricular functions was considered, 37 (35.5\%) patients and $28(26.9 \%)$ patients had left ventricular dysfunction with $\mathrm{LVEF} \leq 50 \%$ and $\mathrm{LVEF} \leq 45 \%$, respectively. No certain definition of patients with REF is present in the literature. LVEF can be accepted as $\leq 50 \%, \leq 40 \%, \leq 30 \%$ and $\leq 45 \%$ in identification of REF in different studies. We also assigned the patients into the REF group according to the most commonly used $\mathrm{LVEF} \leq 45 \%$ value in the literature. A statistically significantly increased LVEF was found when all patients were evaluated (53.9 \pm $\pm 14.6,57.0 \pm 11.4,59.4 \pm 8.4,60.4 \pm 6.8,63.2 \pm$ \pm 3.9 at respectively, baseline, discharge, $1^{\text {st }}, 6^{\text {th }}$, and $12^{\text {th }}$ month; $\left.\mathrm{p}<0.001\right)$. When the REF and PEF groups were evaluated separately, a statistically significantly increased LVEF value $(\mathrm{p}<0.001)$ was found at discharge, $1^{\text {st }}, 6^{\text {th }}$, and $12^{\text {th }}$ month in the REF group whereas LVEF increased in all follow-ups of the PEF group, however this elevation reached a statistical significance only at the $1^{\text {st }}$ month $(p=0.04)$ (Fig. 2). Seventy percent of the patients in the REF group reached normal ejection fraction (LVEF $>50 \%$ ) at the $6^{\text {th }}$ month. Ejection fraction statistically significantly increased in all of the 4 patients with $L V E F \leq 20 \%$, who had increased ejection fraction and reached $>45 \%$ at the end of the $1^{\text {st }}$ month. In the multivariate analyses, there was statistically significant negative correlation between LVEF and STS score, Logistic EUROScore, functional capacity (NYHA), mitral regurgitation and pulmonary artery systolic pressure. But there 
Table 1. Basal characteristics and procedural features.

\begin{tabular}{|c|c|c|c|c|}
\hline Patient characteristics & $\begin{array}{l}\text { All patients } \\
(\mathrm{n}=104)\end{array}$ & $\begin{array}{c}\text { LVEF } \leq 45 \% \\
(n=28)\end{array}$ & $\begin{array}{l}\text { LVEF > 45\% } \\
(n=76)\end{array}$ & $\mathbf{P}$ \\
\hline Male/female & $35 / 69$ & $13 / 15$ & $22 / 54$ & 0.10 \\
\hline Age [year] & $78.2 \pm 7.2$ & $77.4 \pm 7.9$ & $78.5 \pm 6.9$ & 0.50 \\
\hline Body mass index $\left[\mathrm{kg} / \mathrm{m}^{2}\right]$ & $27.9 \pm 7.5$ & $28.1 \pm 11.6$ & $27.8 \pm 5.5$ & 0.89 \\
\hline NYHA class II & 7 & 0 & 7 & 0.08 \\
\hline NYHA class III & 66 & 18 & 48 & \\
\hline NYHA class IV & 31 & 10 & 21 & \\
\hline STS & $7.4 \pm 5.3$ & $11.8 \pm 5.3$ & $5.7 \pm 4.2$ & $<0.001$ \\
\hline \multicolumn{5}{|l|}{ SURTAVI: } \\
\hline Low risk & 10 & 1 & 9 & \\
\hline Moderate risk & 34 & 6 & 28 & 0.03 \\
\hline High risk & 60 & 21 & 39 & \\
\hline EUROScore [\%] & $22.7 \pm 15.8$ & $30.2 \pm 17.9$ & $19.3 \pm 14.1$ & 0.002 \\
\hline \multicolumn{5}{|c|}{ Associated comorbid conditions } \\
\hline Coronary artery disease & $71.1 \%$ & $64.2 \%$ & $73.6 \%$ & 0.41 \\
\hline Hypertension & $81.7 \%$ & $85.7 \%$ & $80.2 \%$ & 0.52 \\
\hline Diabetes mellitus & $25.9 \%$ & $28.5 \%$ & $25.0 \%$ & 0.71 \\
\hline Hyperlipidemia & $45.2 \%$ & $42.8 \%$ & $46.0 \%$ & 0.77 \\
\hline Smoker & $19.2 \%$ & $25.0 \%$ & $17.1 \%$ & 0.36 \\
\hline \multicolumn{5}{|c|}{ Chronic obstructive pulmonary disease: } \\
\hline Mild & $44.2 \%$ & $21.4 \%$ & $52.6 \%$ & \\
\hline Moderate & $30.7 \%$ & $28.5 \%$ & $42.1 \%$ & $<0.001$ \\
\hline Severe & $23.0 \%$ & $50.0 \%$ & $13.1 \%$ & \\
\hline Peripheral arterial disease & $34.6 \%$ & $46.4 \%$ & $30.2 \%$ & 0.12 \\
\hline Atrial fibrillation & $27.9 \%$ & $35.7 \%$ & $25.0 \%$ & 0.28 \\
\hline \multicolumn{5}{|l|}{ Echocardiographic variables } \\
\hline Maximal gradient $[\mathrm{mm} \mathrm{Hg}]$ & $86.2 \pm 21.5$ & $76.5 \pm 19.2$ & $89.8 \pm 21.3$ & 0.005 \\
\hline Mean gradient [mm Hg] & $52.5 \pm 13.7$ & $46.9 \pm 13.7$ & $54.6 \pm 13.2$ & 0.01 \\
\hline Aortic valve area $\left[\mathrm{cm}^{2}\right]$ & $0.62 \pm 0.17$ & $0.60 \pm 0.17$ & $0.63 \pm 0.17$ & 0.42 \\
\hline LVEF [\%] & $53.9 \pm 14.5$ & $33.0 \pm 10.1$ & $61.5 \pm 5.6$ & $<0.001$ \\
\hline Peak SPAP [mm Hg] & $47.8 \pm 13.6$ & $52.3 \pm 15.8$ & $45.9 \pm 12.1$ & 0.04 \\
\hline \multicolumn{5}{|l|}{ Aortic regurgitation: } \\
\hline Low & 17 & 5 & 12 & \\
\hline Moderate & 4 & 2 & 2 & 0.58 \\
\hline Severe & 1 & 0 & 1 & \\
\hline \multicolumn{5}{|l|}{ Mitral regurgitation: } \\
\hline Low & 62 & 19 & 43 & \\
\hline Moderate & 6 & 4 & 2 & 0.009 \\
\hline Severe & 3 & 1 & 2 & \\
\hline Femoral vascular closure & $85.5 \%$ & $85.7 \%$ & $85.5 \%$ & 0.68 \\
\hline \multicolumn{5}{|l|}{ Valve diameter [mm]: } \\
\hline 23 & 59 & 11 & 48 & \\
\hline 26 & 43 & 15 & 28 & 0.01 \\
\hline 29 & 2 & 2 & 0 & \\
\hline Contrast used [cc] & $201.5 \pm 55.7$ & $217.4 \pm 48.6$ & $195.6 \pm 57.3$ & 0.08 \\
\hline $\begin{array}{l}\text { Duration of discharge } \\
\text { after procedure [day] }\end{array}$ & $7.3 \pm 5.3$ & $8.8 \pm 7.1$ & $6.8 \pm 4.4$ & 0.09 \\
\hline
\end{tabular}

NYHA - New York Heart Association, STS — Society of Thoracic Surgeons; SURTAVI — Safety and Efficacy Study of the Medtronic CoreValve ${ }^{\circledR}$ System in the Treatment of Severe, Symptomatic Aortic Stenosis in Intermediate Risk Subjects Who Need Aortic Valve Replacement risk model; LVEF — left ventricular ejection fraction; SPAP — systolic pulmonary artery pressure 


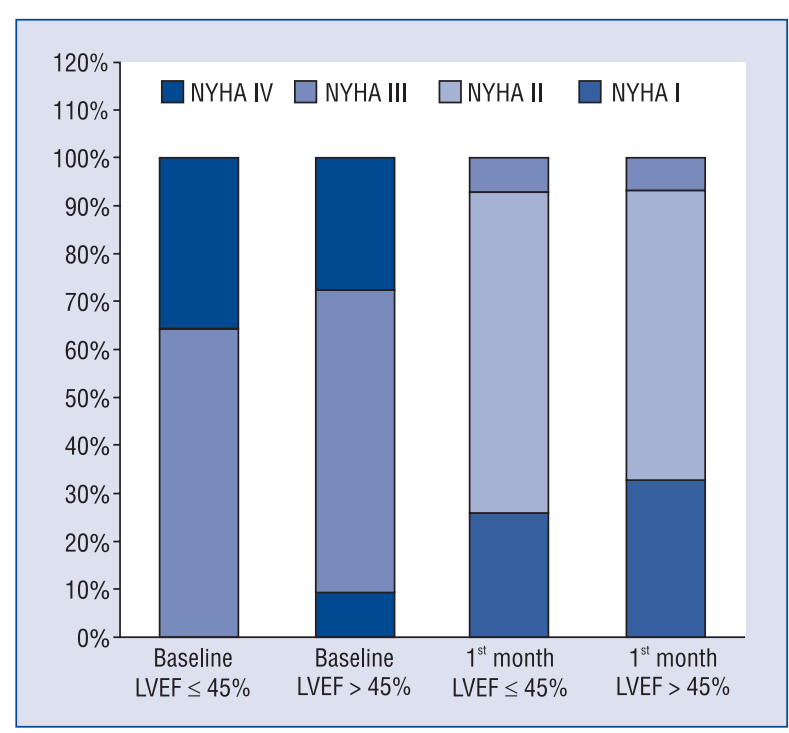

Figure 1. Changes in New York Heart Association (NYHA) functional class at $1^{\text {st }}$ month in patients undergoing transcatheter aortic valve implantation according to left ventricular ejection fraction (LVEF) $\leq 45 \%$ or $>45 \%$.

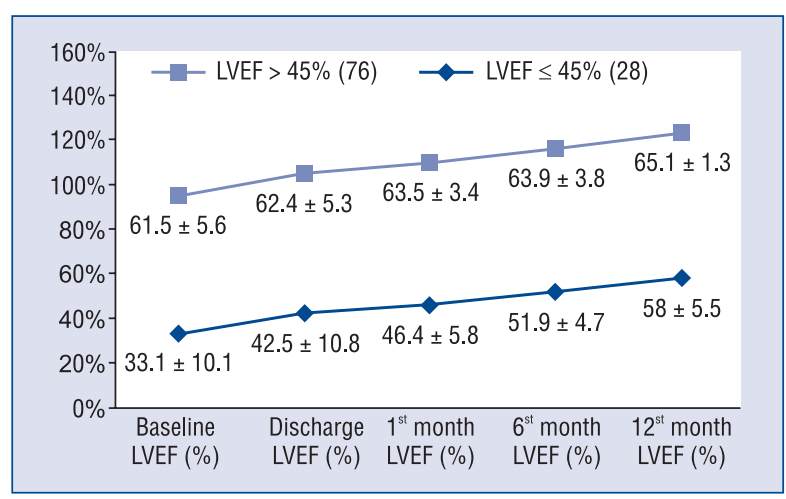

Figure 2. Left ventricular ejection fraction (LVEF) on baseline, improvement during the in-hospital phase, and after a mean follow-up duration of 12 months.

positive correlation between LVEF and mean gradient of aortic valve.

\section{Discussion}

We have shown in our study that TAVI improves left ventricular function in the short and moderate periods. We have proven that LVEF, functional capacity and clinical results of patients with REF improved after TAVI and presented a similar prognosis when compared with patients in the PEF group. Our results also indicate that TAVI may be safe for patients in the REF group; similar to patients in the PEF group, and that TAVI may be unassociated with an increased per procedural risk. It has been shown in the same manner that complication rates of patients in the REF group are comparable to those in the PEF group. Although the incidence of patients with left ventricular dysfunction among all patients $(35.5 \%$ and $26.9 \%$ according to $\mathrm{LVEF} \leq 50 \%$ and $\mathrm{LVEF} \leq 45 \%$, respectively) was high, similar or better clinical results than literature studies have been obtained.

The increased incidence of AS in patients brings many co-morbid risk factors with age. One of these is left ventricular systolic dysfunction. LVEF may decrease due to after load mismatch or intrinsic contractile dysfunction [10]. If the increased LVEF is not caused by AS, aortic valve replacement may provide an improvement in LVEF. Aortic valve replacement is recommended for patients with REF, whether contractile reserve is present or not. DSE was required in only 5 of our patients and contractile reserve was sufficient in these patients. However, impaired left ventricular function is an independent risk factor for early and late mortality after s-AVR $[11,12]$. Postoperative mortalities and complications are also at a high incidence even though left ventricular function improves after successful s-AVR in patients with REF $[12,13]$. TAVI has developed rapidly over the last decade and has become an important treatment option for surgically inoperable or high-risk patients [6, 7]. Some previous as well as new studies indicate improved LVEF after TAVI [8, 14-20]. Especially studies conducted in recent years have evaluated the effect of TAVI on either LVEF or REF in patients. Clavel et al. [8] have compared 200 patients who underwent s-AVR with 83 patients who underwent TAVI for REF (according to LVEF $\leq 50 \%$ ) and have shown a better improvement in the TAVI group in LVEF than the s-AVR group. Bauer et al. [14] found greater improvement at the $7^{\text {th }}$ day in 31 patients with REF (according to LVEF $\leq 45 \%$ ) who underwent TAVI compared to 21 patients receiving s-AVR. They found a similar improvement with respect to LVEF at the $3^{\text {rd }}$ month. Pilgrim et al. [16] in their study obtained results similar to ours, having seen a rapid improvement in 30 patients with REF (according to $\mathrm{LVEF} \leq 30 \%$ ) after TAVI while no difference was found between patients with LVEF $>30 \%$ and the group with REF with respect to complications and mortality. Gotzmann et al. [20] divided patients into four groups based on LVEF (LVEF $>50 \%$ and $\leq 50 \%$ ) and mean gradient ( $>40 \mathrm{~mm} \mathrm{Hg}$ and $\leq 40 \mathrm{~mm} \mathrm{Hg}$ ), as group 1, preserved LVEF/high gradient $(n=86)$, group 2 , preserved LVEF/low gra- 
dient $(\mathrm{n}=27)$, group 3 , reduced LVEF/high gradient $(\mathrm{n}=45)$, and group 4, reduced $\mathrm{LVEF} /$ low gradient $(\mathrm{n}=44)$. This study, which used the CoreValve ${ }^{\circledR}$ valve, found a statistically significant elevation in LVEFs. Nevertheless, this study showed that low gradient and/or low LVEF is associated with poor clinical results. We accepted LVEF $\leq 45 \%$ in our study and obtained successful clinical results in the patients with either REF or low gradient. Early and positive effects of TAVI on LVEF may be explained by several mechanisms. TAVI provides a lower transprosthetic gradient than s-AVR with its wider valve area, thus valve obstruction is completely released and pressure load on the left ventricle is removed. Improvement of LVEF is lower in patients with multivessel coronary artery disease who receive s-AVR concurrently with bypass whereas revascularization could be performed previously, when needed, if TAVI is preferred. Beside these points, other factors related to open surgery during s-AVR, which are irrelevant for TAVI, are ischemia, ischemia/reperfusion, inflammatory response, cardioplegia, surgical trauma, oxidative stress, cardiomyocyte apoptosis due to several factors and contractile dysfunction [21]. The pure percutaneous approach, local anesthesia and non-necessity of vasoactive drug-cardiac arrest-prolonged ventilation during TAVI is a protective characteristic for the pericardium [16]. Since risk scorings (STS, logistic EUROScore) used in patient selection and parameters of left ventricular dysfunction were not evaluated sufficiently, it comes to the forefront that when we consider these data, a scoring system specific for TAVI is necessary. TAVI should also be considered instead of s-AVR in patients with poor parameters of left ventricular function. It is obvious that a value of $\mathrm{LVEF} \leq 20 \%$ is a relative contraindication criteria in the guidelines, which should be reevaluated and patient selection performed based on additional echocardiography criteria (contractile reserve, spackle tracking, left ventricular volume and etc.) and clinical criteria (fragility, functional capacity, age, gender etc.). It can be stated that the procedure can be performed with low complication and mortality rates using carefully conducted patient selection.

\section{Conclusions}

We have shown that an improvement could be obtained using TAVI in left ventricular function and that TAVI could be performed successfully in the REF patient group, producing similar complication rates as the PEF patient group. It can be stated according to this study that selecting TAVI may be necessary instead of s-AVR in patients with left ventricular dysfunction and that left ventricular functions are important parameters to be taken into account in patient selection. Consequently, more comprehensive and randomized studies of these procedures are necessary.

\section{Conflict of interest: None declared}

\section{References}

1. Guidelines on the management of valvular heart disease (version 2012): The Joint Task Force on the Management of Valvular Heart Disease of the European Society of Cardiology (ESC) and the European Association for Cardio-Thoracic Surgery (EACTS). Eur Heart J, 2012; 33: 2451-2496.

2. Iung B, Cachier A, Baron G et al. Decision-making in elderly patients with severe aortic stenosis: why are so many denied surgery? Eur Heart J, 2005; 26: 2714-2720.

3. Bach DS, Siao D, Girard SE, Duvernoy C, McCallister BD, Jr., Gualano SK. Evaluation of patients with severe symptomatic aortic stenosis who do not undergo aortic valve replacement: The potential role of subjectively overestimated operative risk. Circ Cardiovasc Qual Outcomes, 2009; 2: 533-539.

4. Connolly HM, Oh JK, Schaff HV et al. Severe aortic stenosis with low transvalvular gradient and severe left ventricular dysfunction: Result of aortic valve replacement in 52 patients. Circulation, 2000; 101: 1940-1946.

5. Pai RG, Varadarajan P, Razzouk A. Survival benefit of aortic valve replacement in patients with severe aortic stenosis with low ejection fraction and low gradient with normal ejection fraction. Ann Thorac Surg, 2008; 86: 1781-1789.

6. Leon MB, Smith CR, Mack M et al. Transcatheter aortic-valve implantation for aortic stenosis in patients who cannot undergo surgery. N Engl J Med, 2010; 363: 1597-1607.

7. Smith CR, Leon MB, Mack MJ et al. Transcatheter versus surgical aortic-valve replacement in high-risk patients. $\mathrm{N}$ Engl J Med, 2011; 364: 2187-2198.

8. Clavel MA, Webb JG, Rodes-Cabau J et al. Comparison between transcatheter and surgical prosthesis valve implantation in patients with severe aortic stenosis and reduced left ventricular ejection fraction. Circulation, 2010; 122: 1928-1936.

9. Lang RM, Bierig M, Devereux RB et al.; Chamber Quantification Writing Group; American Society of Echocardiography's Guidelines and Standards Committee; European Association of Echocardiography. Recommendations for chamber quantification: a report from the American Society of Echocardiography's Guidelines and Standards Committee and the Chamber Quantification Writing Group, developed in conjunction with the European Association of Echocardiography, a branch of the European Society of Cardiology. J Am Soc Echocardiogr, 2005; 18: 1440-1463.

10. Zile MR, Gaasch WH. Heart failure in aortic stenosis: Improving diagnosis and treatment. N Engl J Med, 2003; 348: 1735-1736.

11. Lund O, Flø C, Jensen FT et al. Left ventricular systolic and diastolic function in aortic stenosis. Prognostic value after valve replacement and underlying mechanisms. Eur Heart J, 1997; 18: 1977-1987.

12. Vaquette B, Corbineau H, Laurent $\mathrm{M}$ et al. Valve replacement in patients with critical aortic stenosis and depressed left ventricu- 
Cardiology Journal 2015, Vol. 22, No. 1

lar function: Predictors of operative risk, left ventricular function recovery, and long term outcome. Heart, 2005; 91: 1324-1329.

13. Kennedy JW, Doces J, Stewart DK. Left ventricular function before and following aortic valve replacement. Circulation, 1977; 56: 944-950.

14. Bauer F, Coutant V, Bernard M et al. Patients with severe aortic stenosis and reduced ejection fraction: Earlier recovery of left ventricular systolic function after transcatheter aortic valve implantation compared with surgical valve replacement. Echocardiography, 2013; 30: 865-870.

15. O'Sullivan CJ, Stortecky S, Heg D et al. Clinical outcomes of patients with low-flow, low-gradient, severe aortic stenosis and either preserved or reduced ejection fraction undergoing transcatheter aortic valve implantation. Eur Heart J, 2013; 34: 3437-3450.

16. Pilgrim T, Wenaweser P, Meuli $\mathrm{F}$ et al. Clinical outcome of high-risk patients with severe aortic stenosis and reduced left ventricular ejection fraction undergoing medical treatment or TAVI. PLoS One, 2011; 6: e27556.
17. Webb JG, Pasupati S, Humphries K et al. Percutaneous transarterial aortic valve replacement in selected high-risk patients with aortic stenosis. Circulation, 2007; 116: 755-763.

18. Clavel MA, Webb JG, Pibarot $\mathrm{P}$ et al. Comparison of the hemodynamic performance of percutaneous and surgical bioprostheses for the treatment of severe aortic stenosis. J Am Coll Cardiol, 2009; 53: 1883-1891.

19. Bauer $\mathrm{F}$, Eltchaninoff $\mathrm{H}$, Tron $\mathrm{C}$ et al. Acute improvement in global and regional left ventricular systolic function after percutaneous heart valve implantation in patients with symptomatic aortic stenosis. Circulation, 2004; 110: 1473-1476.

20. Gotzmann M, Rahlmann P, Hehnen T et al. Heart failure in severe aortic stenosis: Prognostic impact of left ventricular ejection fraction and mean gradient on outcome after transcatheter aortic valve implantation. Eur J Heart Fail, 2012; 14: 1155-1162.

21. Connolly HM, Oh JK, Orszulak TA et al. Aortic valve replacement for aortic stenosis with severe left ventricular dysfunction. Prognostic indicators. Circulation, 1997; 95: 2395-2400. 